\title{
Smart, Connected, and Autonomous Automobiles - the impact
}

\section{Prof. Gary J. Mullett, Springfield Technical Community College}

Gary J. Mullett, a Professor of Electronics Technology and Co-Department Chair, presently teaches in the Electronics Group at Springfield Technical Community College (STCC) located in Springfield, MA. A long time faculty member and consultant to local business and industry, Mullett has provided leadership and initiated numerous curriculum reforms as either the Chair or Co-Department Chair of the four technology degree programs that constitute the Electronics Group. Since the mid-1990s, he has been active in the NSF's ATE and CCLI programs as a knowledge leader in the wireless telecommunications field. A co-founder of the long running National Center for Telecommunications Technologies (then the ICT Center) located at STCC, Mullett also played a principle role in the development of the innovative and long running Verizon NextStep employee training program. The author of two text books, Basic Telecommunications - The Physical Layer and Wireless Telecommunications Systems and Networks, Mullett did both his undergraduate and graduate work (in Remote Sensing) in the ECE Department at the University of Massachusetts at Amherst where he also taught the undergraduate sequence of courses in electromagnetics. He has presented at numerous local, regional, and national conferences and also internationally on telecommunications and wireless topics and on the status of the education of electronics technicians at the two-year college level. His current interests are: the development of novel and innovative systemslevel approaches to the education of technicians, applications of the emerging field of wired and wireless networked embedded controllers and sensor/actuator networks, and cyber-physical system applications in the context of the Internet of Things (IoT). 


\title{
Smart, Connected, and Autonomous Automobiles - the impact on two-year college technical education
}

\begin{abstract}
Although most of the population cannot afford a new automobile every new model year, the auto makers use mass media to advertise their new vehicles each year and to introduce the public to the new features of their products. It should be obvious to even the casual observer that today's automobiles have never been as intelligent or technologically sophisticated as they presently are. In fact, automobiles are in the process of morphing into super-computers/robots on wheels. It is widely predicted that in a few short years autonomous cars will be in mass production. As they become an appreciable fraction of the automotive fleet, many believe that automotive transportation will forever change for the better. The most important change will involve vehicle safety but many have predicted a whole multitude of other sociological changes to human behavior involving automotive transportation. Today, the automobile manufacturers advertise advanced driver assistance systems (ADAS) like self-braking or collision avoidance systems. Enabled by networked embedded controllers and complex sensors and actuators, ADAS systems are becoming standard equipment on an automakers fleet and not just the high-end models. In the near future, using these enabling technologies, even more sophisticated sensors, and emerging wireless networking technologies the automobile will become a true cyber-physical system aware of its surroundings and eventually capable of autonomous operation.

Presently, formal education of these advanced technology enablers is woefully lacking at vocational (K-12), post-secondary technical schools, and two-year colleges that teach automotive technology. Furthermore, most colleges that offer electrical/electronic engineering technology (EET) education at the two-year college level, as of yet, do not have educational courses or programs to teach this new technology if they were called upon to fulfill the need. The enabling technologies for these ADAS systems are inter-disciplinary in nature. Computer networking for automobiles, embedded controllers, wireless networking, radar and LiDAR are not common topics found in typical EET programs, let alone automotive technology programs that still tend to focus on traditional mechanical technologies. Truly autonomous automobiles will also need an additional support infrastructure that will allow vehicle-to-vehicle (V2V) communications, as well as, vehicle-to-infrastructure (V2I) or vehicle-to-roadside (V2R) wireless networking. There will need to be an effort made to supply technicians with the skill sets needed to install, evaluate, maintain, and up-grade these advanced automotive systems and support infrastructure as they are presently being manufactured and envisioned for the future. This paper will attempt to present a "road map" to a future curriculum that will satisfy the needs of this rapidly emerging transformation in automotive transportation technology.
\end{abstract}

\section{Introduction:}

For the past four decades, automobile manufacturers have been incrementally adding electronics to their vehicles. Early on, the reason for this was quite simple. Mechanical parts wear out and 
therefore require preventive maintenance, repair, and/or complete replacement. Mechanical points used in distributor based legacy ignition systems (with a life-span of roughly 12,000 15,000 miles) were eventually replaced by electronic ignitions that have the benefit of much longer life-spans. Presently, the distributor-less ignitions in today's vehicles may never need servicing during the life-time of the vehicle. Furthermore, as time went on, with the advent of on-board computers and sensors for camshaft and crankshaft position, the vehicle's electronic control module (ECM) now controlled the ignition spark and timing and did it better than the old distributor based systems. With the introduction of emissions and gas mileage standards for automobiles, manufacturers initially contrived improvements through mechanical designs (e.g. exhaust gas recirculation, catalytic converters, etc.). Eventually electronic based innovations and improvements like electronic fuel injection (EFI) systems and exhaust $\mathrm{O}_{2}$ sensors combined with electronically controlled transmissions and improved ECMs provided the enhancements to lower emissions and increase mileage. As the amount of automobile on-board electronics continued to rise with the addition of other microcontrollers (known in the industry as electronic control units or ECUs), the need for improved vehicle diagnostics and some form of vehicle communications network to implement the diagnostic system and coordinate the operations of the various control systems arose. The result was the initial OBD (on-board diagnostic) system and the venerable CAN (controller area network) bus. About the same time as these innovations, vehicle safety became an international concern and eventually safety legislation in the United States mandated the use of front airbags in all passenger cars and light trucks built after September $1,1998^{1}$. This safety innovation was achieved through the use of a sophisticated electronic crash sensor and the airbag electronic control unit. This particular use of electronics signaled the start of the era of "passive” or "passive reactive” passenger safety protection. That is, providing some form of passenger protection after an accident or collision has happened.

\section{Today's Vehicle Technology:}

Today's automobiles may have upwards of 50 to 75 microcontrollers on board, the ECM may run several to many million lines of code, and approximately one third or more of the vehicle's cost may be attributed to the on-board electronic control systems. By 2030 this cost share ${ }^{2}$ is expected to rise to 50\%! One may categorize automotive electronics into five broad areas of application: Infotainment, Body Electronics, Chassis Electronics, Powertrain Electronics, and Advanced Driver Assistance Systems (ADAS) ${ }^{3}$. Furthermore, we have entered the era of hybrid, electric, and alternative fuel vehicles which requires even more complex and high-power electronic controls to implement these technologies effectively. Let's briefly survey these broad application areas. Automotive Infotainment systems are evolving to encompass the concept of the "connected vehicle”. Besides the classic in-car radio entertainment system with CD players and MP3 compatibility, today's vehicles are able to receive satellite entertainment and global positioning system (GPS) signals, as well as, the traditional terrestrial AM and FM stations. Vehicles equipped with "OnStar" or similar type plans utilize the cellular telephone system to communicate and now provide emergency, security, navigation, and Internet connectivity ${ }^{4}$. In car Wi-Fi hot spots are now available and most cars come with an information cluster display 
(LCD screen) that integrates most of the infotainment control functions into one central area and also displays navigation and on-board camera information. In the near future, the automobile will be wirelessly connected to the "cloud" which will allow for navigation, safety and traffic updates, as well as, passenger connectivity for Internet access and streaming services. Vehicle to anything (V2X) connectivity is another matter and will be discussed in the context of autonomous cars. Body electronics consists mainly of the vehicle's data bus networks, rearview mirror features, doors, locks, and windows, and occupant safety features. There are several different types of automotive vehicle data buses in use and they are typically differentiated by their speed and purpose. The CAN bus standard has been updated to version 2.0 and FlexRay, LIN, and MOST bus protocols have all been revised to deal with new technologies and speed requirements. Many observers of the automotive industry believe that the Ethernet networking standard will eventually need to be adopted as the automobile becomes more connected to the cloud and additional cloud based applications are implemented (i.e. traffic control, vehicle prognosis, etc. $)^{5}$. As of today, only the Tesla car company has adopted the Ethernet standard for its in-vehicle data network. Also, it should be noted that the on board diagnostic system has been upgraded and is now known as OBDII. Chassis electronics refers to vehicle speed control systems, anti-locking braking systems (ABS), adaptive suspension systems, and electronic stability control (ESC). All of these systems use "smart” electronic control systems that take sensor input signals and under program control modify the vehicle's operation to protect the occupant from hazardous or potentially unsafe conditions by improving the vehicle's stability or traction or traffic speed. In the United States, ESC was mandated for all passenger vehicles built after 2012 by the National Highway Transportation Safety Administration (NHTSA) ${ }^{6}$. Large trucks will be required to have ESC by 2017. Powertrain electronics basically refer to the initial vehicle electronic control functions of the early ECMs. Engine and automatic transmission controls that reduce emissions and improve engine efficiency. In the case of hybrid and electric vehicles, these controls provide an interface between the gasoline powered engine and the electric engine (motor) in a hybrid, and provide control of all functions for totally electric vehicles. Additionally, the operation of the continuously variable speed (CVS) transmission and vehicle battery charging are also controlled by the powertrain electronics.

The last category of automotive electronics is that of advanced driver assistance systems (ADAS). What is different about these systems is that they are no longer passive safety systems. They are all active safety systems that are implemented to protect the vehicle's occupants by preventing accidents or collisions (the chassis electronic systems detailed earlier also fit this category but usually are not labeled as ADAS systems). What further differentiates these systems is that they belong to new class of electronic control systems known as cyber-physical systems (CPS). Cyber-physical systems are defined as embedded control systems that are tightly coupled to the real (physical) world and require critical timing to perform their function. Different automobile manufacturers offer various versions of these systems which consist of: collision warning or collision imminent notifications, lane-change, blind-spot warning, lane-departure warning, backup assist, parking assist, self-parking, night vision, heads-up displays, 
pedestrian/wild life/obstacle detection, automatic/autonomous emergency braking (AEB) systems, and finally autonomous (self-driving) vehicle operation. These systems utilize sophisticated advanced remote sensing technologies like cameras, ultrasonic sensors, radar, and LiDAR (a laser beam enabled form of radar). Additionally, there are initiatives in the planning stages and active trials of what is known as V2X (vehicle-to-anything) wireless technology systems. V2X consists of several variations: V2V or vehicle-to-vehicle, V2R or vehicle-toroadside, and V2I or vehicle-to-infrastructure wireless links. In these various communications scenarios, vehicles form ad hoc wireless networks and share driving information (lane change intention, braking, acceleration, direction, turning, etc.) with other nearby vehicles or obtain traffic or other types of information from roadside beacons or the surrounding physical infrastructure (e.g. traffic controllers at intersections, highway signage, etc.). As was mentioned previously, the modern car qualifies as a cyber-physical system since it has embedded control that is tightly coupled locally and has critical timing associated with the attendant control functions. To implement these ADAS systems, the automobile uses a combination of sensors to learn about its surroundings and uses data fusion and what some would call artificial intelligence (AI) to make time critical decisions about the safest course of action. When additional wireless communications with nearby vehicles and physical structures becomes available, the automobile will be able to be "aware" of its local surroundings and make decisions that will protect the safety of its passengers. Thus, becoming an "intelligent” cyber-physical system that will eventually transform the concept of passenger vehicle transportation as the number of vehicles with this capability increases. Of course, practically speaking, until all vehicles have compatible, standardized systems and smart support infrastructure is in place, the ultimate benefits of these collision avoidance systems will not be totally realized. However, various large automotive vehicle manufacturers have set time lines for the introduction of vehicles with these capabilities so it is no longer "if" but now "when will" we see the benefits of self-driving or autonomous automotive technology.

\section{The Problem:}

Traditionally, automotive maintenance and repair was very much a mechanical enterprise and was predicated on observation of vehicle performance and/or scheduled servicing tied to miles driven (i.e. oil changes, tune-ups, tire rotations, brake pad replacement, etc.). Replacement of worn out or failed parts (minor or major in cost or function) typically brought the vehicle back into service. Until recently, automobile vehicle manufacturers have dealt with the evolving electronic aspect of automobiles in a fairly standard fashion when considering maintenance and repair protocols. When an electronic system or a sensor that monitors vehicle operation fails or indicates a drivability or system control problem, a code (or codes) is set by the system and depending upon its severity or frequency of occurrence an annunciator on the dash will light up (the infamous "Check Engine Light") to warn the operator. The code(s) will be available on the OBD interface for diagnosing and servicing. The cure for the problem is for the repair technician to replace the sensor or use the code or codes to troubleshoot the problem and narrow the cause of the problem down to a specific part or system and to replace that part or system (i.e. swap out 
the bad part(s)). Unfortunately, if the problem is intermittent or possibly caused by a faulty wiring harness or by an evolving mechanical defect, solving the mystery of what is causing the problem can become extremely vexing. To determine the cause of various drivetrain faults the automotive industry has had to develop and employ more sophisticated diagnostic "scan" tools that monitor real time vehicle operation and special monitoring tools for the various in-vehicle network buses to detect faults related to their operation. In response to the increased amount of electronics in vehicles, many automotive technician programs now include basic electricity/ electronics theory as part of their curriculum.

Examination of a typical two-year college automotive technology program reveals that the usual curriculum provides a basic course or courses that serve as an introduction to basic electrical theory (DC and AC) and possibly additional courses that introduce some fundamentals of electronics and electronic controls. Often times, these courses are grounded in the context of the automobile's technology. For example, Macomb Community College located in the heart of automobile manufacturing country in Warren, MI lists three "electrical/electronic" courses in its popular Automotive Technology, AAS degree program curriculum:

\begin{tabular}{|l|l|}
\hline $\begin{array}{l}\text { Automotive } \\
\text { Electrical Systems }\end{array}$ & $\begin{array}{l}\text { This course provides an introduction to the fundamentals of electricity. This course will } \\
\text { focus on a working knowledge of the basic automotive electrical systems, components, } \\
\text { and general diagnosis. Emphasis is placed on locating and interpreting specifications, } \\
\text { electrical tests, and correct use of meters and test equipment }\end{array}$ \\
\hline $\begin{array}{l}\text { Automotive } \\
\text { Electronics }\end{array}$ & $\begin{array}{l}\text { This course will focus on the operation and application of vehicle's electronic controlled } \\
\text { circuitry and locating the components on the vehicles. It introduces semi-conductors } \\
\text { and microprocessors to facilitate the understanding of components used on automotive } \\
\text { electronic systems. On-bench and on-car practical exercises provide additional } \\
\text { substance to the course and reinforce classroom learning }\end{array}$ \\
\hline $\begin{array}{l}\text { Advanced } \\
\text { Automotive } \\
\text { Electronics }\end{array}$ & $\begin{array}{l}\text { This course is designed to provide the student with the latest state-of-the-art } \\
\text { technology as related to automotive electronics. This course will focus on such criteria } \\
\text { as: test equipment for solid state circuitry, electronic cruise control, level ride, travel } \\
\text { assist and driver information centers, keyless entry system, and sound systems } \\
\text { including service. On-bench and on-car practical exercises are featured with the } \\
\text { classroom activities. }\end{array}$ \\
\hline
\end{tabular}

Table 1

As can be seen in the table, these three courses present the needed electricity/electronics concepts in the context of a modern automobile. However, the depth of knowledge is limited and there is little information about the on-board communications networks or advanced concepts of embedded control. Furthermore, details of recently implemented ADAS systems is almost non-existent.

A fundamental principle of the endeavor of evaluating, maintaining, and repairing technology systems, including today's very sophisticated and technologically advanced automobiles, is that the more the technician understands about how the system works the easier it is to diagnose and repair malfunctions that it might be experiencing. Therefore, a fundamental precept of technical education programs is that students are taught the principles and concepts of operation of the 
various technology systems germane to the particular technology field. Most automotive technology programs follow this model by providing individual courses on: Brakes \& Suspension, Drivability \& Diagnosis, Starting \& Charging Systems, Powertrain Systems, Air Conditioning, Ignition \& Emissions Systems, Fuels systems, Engines, and Automotive Electronics (see Table 1 above). Most of the evaluation of electronic control system operation is left to the OBDII system or more sophisticated scan tools. To some extent, this is perfectly acceptable since the vast majority of the faults experienced by a modern automobile can be detected and repaired using this protocol. However, nearly everyone has heard of a horror story or two about multiple replacements of a vehicle's ECMs without a resolution of the problem or continuing trips back to the dealer or repair garage without a permanent solution to a drivability problem or some other aspect of the vehicle's operation. Without a change in what is taught to students in the technology fields (including automotive technology programs), this fairly rare occurrence of an unsolvable problem is only going to become more commonplace.

This "electronic-ization" of today's automobiles as characterized by Cisco has brought the automotive industry to an inflexion point. According to Cisco the auto industry is converging towards high tech, another innovation-based industry ${ }^{8}$. To be sure, this problem has struck a chord with various technology educators at the two-year college level. Returning to Macomb Community College, the college has received a National Science Foundation (NSF) Advanced Technology Education (ATE) grant to host a center of excellence known as the Center for Advanced Automotive Technology (CAAT). Through the Center's activities, CAAT has facilitated the development of curriculum suitable for two-year college level programs and certificates about various emerging automotive technologies. Various courses on hybrid, electric, and fuel-cell vehicles exist and a course on automated, connected, and intelligent vehicles is under development ${ }^{9}$. The CAAT has done a great deal of beneficial work to start on a solution to the problem of the rapid integration of advanced electronics technology into today's automotive vehicles but will it be enough is the question and is there a need for legacy EET programs to become involved in the solution.

\section{The Big Picture}

In this author's opinion, unless needed change occurs in technology education, there is an overarching disruptive technology that will affect many technology disciplines and will make it difficult to provide the skill sets needed by technicians in many fields, not just automotive technology. This phenomenon is known as the Internet of Things or IoT. For many years now, observers of technology have noted several emerging technologies implemented with electronics that are converging together to enable new and society changing technology applications ${ }^{10-21}$. These enabling technologies include but are not limited to networked embedded controllers (known as ambient intelligence), wireless data networking, advanced sensor and actuator networks, and a ubiquitous Internet. One of the most disruptive (in a good way) and potential society changing technologies that will arise out of the IoT paradigm is the ability to design and deploy cyber-physical systems. These systems will enhance the efficiency, safety, and security of 
most human endeavors - whether it be commerce, work, education, or play, and they will have the real capability to change almost every aspect of mankind's interaction with the environment. Recent advertisement campaigns by IBM (The Smarter Planet) and Cisco (Internet of Everything) and technology initiatives by Google, Microsoft, and others indicate that these technology companies believe that IoT applications will drive the market for the foreseeable future. However, these enabling technologies tend not to be taught by two-year college technology programs since they are inter-disciplinary in nature and presently tend to belong exclusively to one area or another. Computer networking is a topic not typically taught in electrical/electronic type programs even though we are moving towards the era when everything electronic has an IP address and is therefore networkable and in many instances reconfigurable via the Internet. Electronic communications may be taught in EET type programs but usually not wireless networking because that belongs to the IT world - but they don't teach it! It least not at the physical/hardware layer. Radar and LiDAR technologies are rarely emphasized in EET programs. Embedded controllers might be taught in EET programs but not networked applications with critical timing. Wireless networking technologies are posed to move into the millimeter frequency bands (i.e. IEEE 802.11ad). What percentage of faculty of EET type programs have experience with millimeter waves or equipment that can even display the signals? Very little curriculum exists about these topics and to further complicate matters the rate of change in these areas is difficult to keep up with. Therefore, faced with a lack of courses and/or curriculum in these inter-disciplinary areas many technology fields will find it difficult to hire employees with the skill sets necessary to maintain and repair their products and systems as new applications of IoT are deployed.

Let's return to automotive technology and the effect of this emerging technology on this area. An overview of the new ADAS technologies to be deployed has already been given. Automobile manufacturers have already agreed to implement AEB system technology (in 99\% of passenger vehicles) by the 2022 model year ${ }^{22-23}$. Google has been testing a fleet of self-driving cars for years and Ford Motor Company has announced that they are targeting fully autonomous cars for ride sharing applications by $2021^{24}$ as have others like General Motors and Tesla. Toyota, Honda, Mercedes-Benz, and most other major manufacturers have also committed to joining the race to produce autonomous vehicles but they are less definitive in their expectations of when their vehicles will actually be ready for market. The technology employed to make autonomous cars a reality is extremely complex, cutting edge, and not thoroughly vetted at this time. Besides the complex hardware there are challenges in the remote sensing technologies, the aspects of artificial intelligence used to make decisions, the reliability of wireless network links with difficult and constantly changing propagation characteristics, robotics functions adapted to automobiles, and the list goes on. How much self-testing and self-diagnosing of the various hardware sub-systems and components will be possible is problematic and when the vehicle is actually in the driving environment it is unknown whether or not operational models will predict proper operation. How will the OBDII system or its successor(s) provide useful diagnostic 
information about the on-board cyber-physical systems where critical timing is crucial for correct operation. How will repair shops determine correct operation of the ADAS systems. Right now, there are many more questions than answers. Two facts are for certain however, the skill sets needed to deal with this technology are not presently being taught to students in automotive technology programs. For that matter, most of this type of technology is not being taught presently by its originating field - electronics technology. Secondly, this technology is coming faster than most believe is possible. The automobile of the future (i.e. the next decade!) will be a super-computer on wheels as far as on-board technology is concerned and will serve as a vehicular robot which will provide driverless, safe transportation.

\section{A Possible Solution:}

The under-pinning technologies of IoT applications across various fields of technology are depicted below in Figure 1. It really doesn't matter if one talks about e-health care applications,

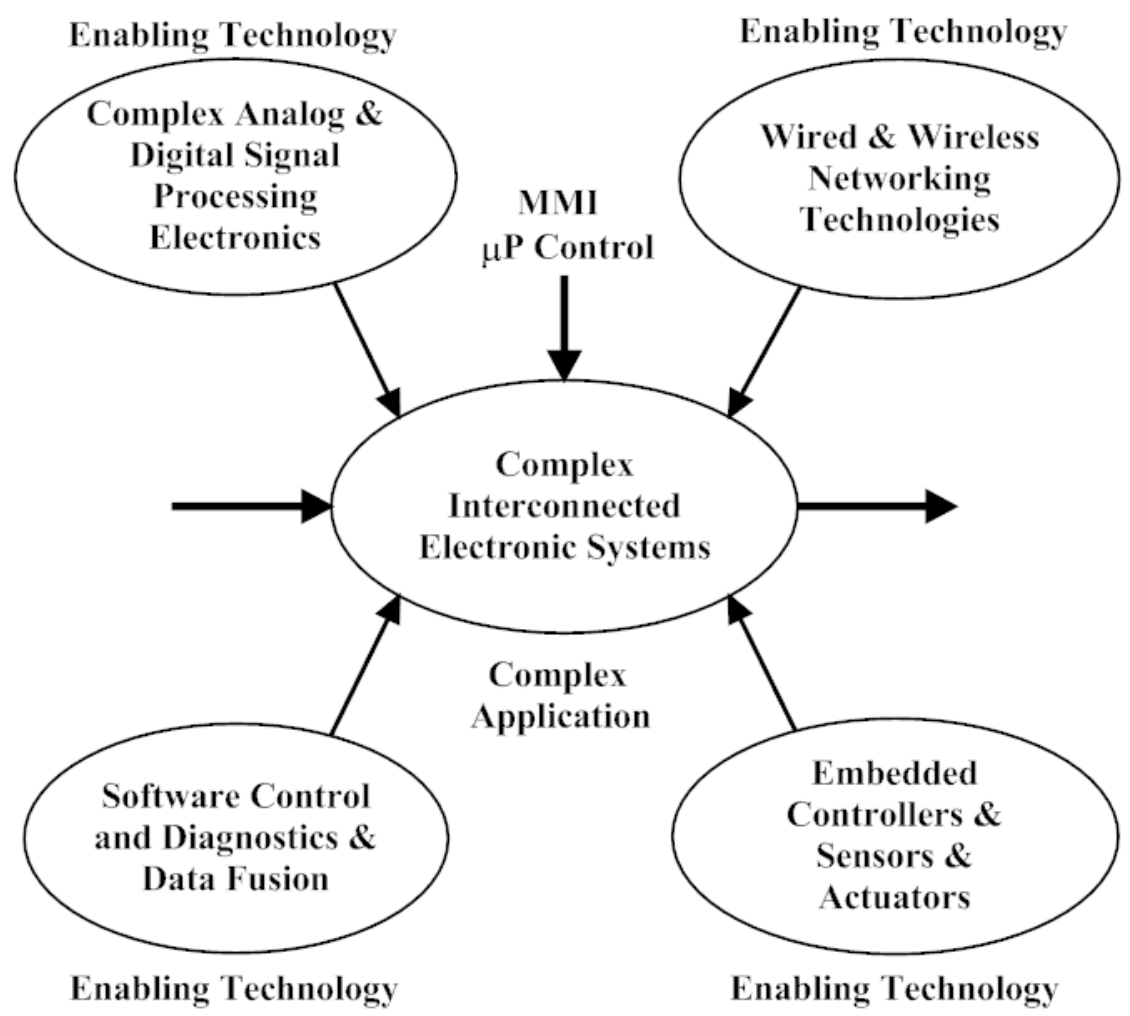

Figure 1 - The underlying technologies of the Internet of Things ${ }^{25}$

the Smart Grid, building automation, or autonomous vehicles. The relevant technologies are: basic electronics, networking (wireless and hard-wired), embedded controllers, advanced sensors and actuators, and software. The technician that will deal with this type of technology will still need to know some fundamental concepts in DC and AC theory, basic electronics from a systems perspective, and system interfacing. In this context, when talking about a system, it should be 
understood that one is referring to systems germane to the particular field of endeavor (e.g. powertrain systems, ABS, ESC, etc. for the automotive area). Additionally, the basic operation of embedded controllers and how one interfaces to and programs these devices should also be considered a fundamental skill. Topics like basic networking theory (i.e. TCP/IP) for both wired and wireless networking technologies with applications to the situation of interest will be a necessary skill in the intelligent or smart environment created by sensor networks. For automotive technology, this necessitates learning about the special network buses used by the automotive industry and their protocols. It is logical that sensor and actuator theory should also be part of the technician's skill set and not be limited to mechanical operations but should include remote sensing devices or at a minimum the basic theory of their operation. Certainly, basic system software concepts and security become important when dealing with today's technology. Software and PC skills become an additional tool set of this new type of technician augmenting or replacing much of the testing hardware of the past as their most important tools of the trade. As pointed out earlier, many of these topics just mentioned are presently under the domain of different technology fields. What is being suggested here is that the skill sets needed by the technician of the future need to be taught across numerous technology fields. As complex, networked, electronic systems become deployed by different technology fields there begins to becomes some common skill sets needed by each field. Ideally, the course material would be developed by the electronics technology folks in conjunction with the particular application technology field. In this scenario, the EET program becomes a special type of applications centered "service department" for other technology areas.

\section{Conclusion:}

Electronics technology has always been one of the fastest changing technologies and today continues to morph and evolve allowing for innovations and new applications that were a short time ago considered science fiction but now are becoming tomorrow's reality. Today the automotive industry's use of electronics in passenger vehicles is on the threshold of another paradigm shift with the advent of ADAS systems that implement cyber-physical systems that will make automobiles safer and eventually autonomous. As surely as this evolution of the automobile will take place it will also have an ever-increasing effect on automotive technology education. The automotive technician of the next decade will need to be able to: evaluate complex electronic system operation and performance through their knowledge of the system's intended function and a further knowledge of its acceptable parameters of operation. The evaluation of system operation by this technician most likely will be facilitated through diagnostic software and sophisticated built-in-self-test (BIST) at both the sub-system and system level. Forward-looking automotive technology programs that embrace the teaching of automotive electronics from a systems approach, craft their curricula carefully to be systems centric and hands-on, and embrace new teaching techniques that give their students the skills to become life-long learners will be preparing their students for the technology of the future and their continued relevance in that field. 
Other fields of technology will also be impacted by this most recent convergence of technologies and availability of ubiquitous communications. Any type of technology that involves some type of physical infrastructure or conveyance will likely be fair game for the addition of networked, ambient, intelligence, and/or cyber-physical systems to improve its operation. How will this transition in the curricula of technology programs be achieved? In this author's opinion, the creation of advanced certificates is most likely the least painful way to proceed. After a student has earned a degree in the particular technology field, taking additional courses about the emerging technologies of smart IoT applications in that field would lead to a certificate in advanced automotive systems for example or an advanced certificate in some other technology field.

\section{Bibliography}

1. http://www.afdc.energy.gov/pdfs/2457.pdf

2. https://www.statista.com/statistics/277931/automotive-electronics-cost-as-a-share-of-total-car-costworldwide/

3. Forty-Year Review of Automotive Electronics: A Unique Source of Historical Information on Automotive Electronics, by William J. Fleming, IEEE Vehicular Technology Magazine, Volume 10 Number 3, Pages 80-90, September, 2015

4. https://www.onstar.com/us/en/home.html

5. Personal communications with Dr. Harita Joshi re: Vehicular Networks, September 18, 2016

6. https://www.nhtsa.gov/sites/nhtsa.dot.gov/files/fmvss/ESC_FR 03_2007_0.pdf

7. http://ecatalog.macomb.edu/preview_program.php?catoid=9\&poid=1934\&returnto=326

8. http://www.cisco.com/c/dam/en_us/about/ac79/docs/mfg/Electronics-ization_in_Automotive.pdf

9. http://autocaat.org/uploadedFiles/Content/Educators/Seed_Funding/Seed\%20Funding\%20Matrix.pdf

10. The Disappearing Associates Degree Program in Electronics Technology, by Louis E. Frenzal Jr. , Proceedings of the 2003 American Society of Engineering Educators Annual Conference and Exposition, Nashville, TN

11. The 2010 Gigascale Imperative: Why the instruction of electronics technology must change!, by Gary J. Mullett, Proceedings of the 2005 American Society of Engineering Educators Annual Conference and Exposition, Portland, OR

12. Are the Electronics Technology Departments of Today, Destined to Become Academic Service Departments of Tomorrow?, by Gary J. Mullett, 2006 SAME-TEC Conference, Santa Fe, NM, available on the SAMETEC web site, Conference Proceedings 2006: www.same-tec.org

13. Are Today's Electronics Technology Programs Doomed to Extinction or is their Mission Changing?, by Gary J. Mullett, Proceedings of the 2007 American Society of Engineering Educators Annual Conference and Exposition, Honolulu, HI

14. The New Electronics Technology - Circa 2015, by Gary J. Mullett, Proceedings of the 2009 American Society of Engineering Educators Annual Conference and Exposition, Austin, TX

15. Its 2010 and the new Electronics Technology Paradigm is Emerging, by Gary J. Mullett, Proceedings of the 2010 American Society of Engineering Educators Annual Conference and Exposition, Louisville, KY

16. Smart Grid, Cleantech, Sensor Networks Come of Age, by Gary J. Mullett, Proceedings of the 2010 American Society of Engineering Educators Annual Conference and Exposition, Louisville, KY

17. Intelligent Infrastructure Systems and the Technician, by Gary J. Mullett, Proceedings of the 2011 American Society of Engineering Educators Annual Conference and Exposition, Vancouver, B.C., Canada

18. Teaching Networked Embedded Control At the Two-Year College Level, by Gary J. Mullett, Proceedings of the 2012 American Society of Engineering Educators Annual Conference and Exposition, Austin, TX

19. The Internet of Things (IoT) will create the need for the Cyber-Physical Systems Technician, by Gary J. Mullett, Proceedings of the 2014 American Society of Engineering Educators Annual Conference and Exposition, Indianapolis, IN 
20. The creation of a Biomedical Technology Program for the 2020s, by Gary J. Mullett, Proceedings of the 2015 American Society of Engineering Educators Annual Conference and Exposition, Seattle, WA

21. Teaching the Internet of Things (IoT) using Universally Available Raspberry Pi and Arduino Platforms, by Gary J. Mullett, Proceedings of the 2016 American Society of Engineering Educators Annual Conference and Exposition, New Orleans, LA

22. http://www.automotiveworld.com/analysis/regulating-safety-semi-autonomous-world-us-aeb-agreement/

23. http://autoweek.com/article/technology/will-autonomous-braking-come-ahead-federal-mandate

24. http://www.usatoday.com/story/tech/news/2017/02/10/ford-bets-1b-self-driving-car-startup/97745230/

25. Same as 20 